\title{
A 38-year-old Man with Left Knee Pain
}

\author{
Benjamin K. Potter MD, Sheila C. Adams MD, \\ Mark J. Kransdorf MD, H. Thomas Temple MD
}

Published online: 29 January 2009

(C) The Association of Bone and Joint Surgeons 2009

\section{History and Physical Examination}

A 38-year-old man presented with a 2.5-month history of spontaneous onset of dull, aching left knee pain localized over the proximal tibia and anteromedial knee. The pain was aggravated by activity, improved by rest, and absent at night. It was not relieved by nonsteroidal antiinflammatory drugs or mild narcotic analgesics, and he subsequently was referred for orthopaedic evaluation. The patient denied any constitutional symptoms such as fever, chills, night sweats, fatigue, or recent weight loss.

Physical examination of the left lower extremity revealed no skin abnormalities, palpable masses, or lymphadenopathy.

Each author certifies that he or she has no commercial associations (eg, consultancies, stock ownership, equity interest, patent/licensing arrangements, etc) that might pose a conflict of interest in connection with the submitted article.

Each author certifies that his or her institution has approved the human protocol for this case report, that all investigations were conducted in conformity with ethical principles of research, and that informed consent was obtained.

B. K. Potter, S. C. Adams, H. Thomas Temple Department of Orthopaedics, University of Miami Miller School of Medicine, Miami, FL, USA

\section{B. K. Potter $(\bowtie)$}

Integrated Department of Orthopaedics and Rehabilitation, Walter Reed Army Medical Center, 6900 Georgia Avenue, NW, Building 2A, Room 205, Washington, DC 20307, USA

e-mail: Benjamin.Potter@amedd.army.mil

B. K. Potter

Department of Surgery, Uniformed Service University of Health Sciences, Bethesda, MD, USA

\section{J. Kransdorf}

Department of Radiology, Mayo Clinic, Jacksonville, FL, USA
Neurologic examination was normal. The left knee was stable with no joint line tenderness but had decreased range of motion from $0^{\circ}$ to $70^{\circ}$ and a moderate effusion. There was mild tenderness to palpation over the proximal medial tibia.

Imaging studies included radiographs (Fig. 1) and MRI (Fig. 2).

Based on the history, physical examination, and imaging studies, what is the differential diagnosis?

\section{Imaging Interpretation}

Anteroposterior (Fig. 1A) and lateral (Fig. 1B) radiographs of the left knee revealed a radiolucent lesion located in the medial aspect of the proximal tibial metaphysis and extending into the epiphysis with no evidence of internal mineralization. The lesion was eccentric with ill-defined, geographic 1C margins laterally [10]. There was no periosteal reaction or violation of the articular surface or medial tibial cortex.

Coronal T1-weighted (Fig. 2A) and STIR (Fig. 2B) MR images showed a mildly heterogeneous lesion with hypointense to isointense signal relative to muscle on the T1 images and increased signal relative to fat on STIR imaging. There was extensive abnormal signal extending medially into the adjacent soft tissues in addition to mildly increased signal in the marrow adjacent to the lesion.

\section{Differential Diagnosis}

Giant cell tumor

Benign fibrous histiocytoma

Chronic osteomyelitis 

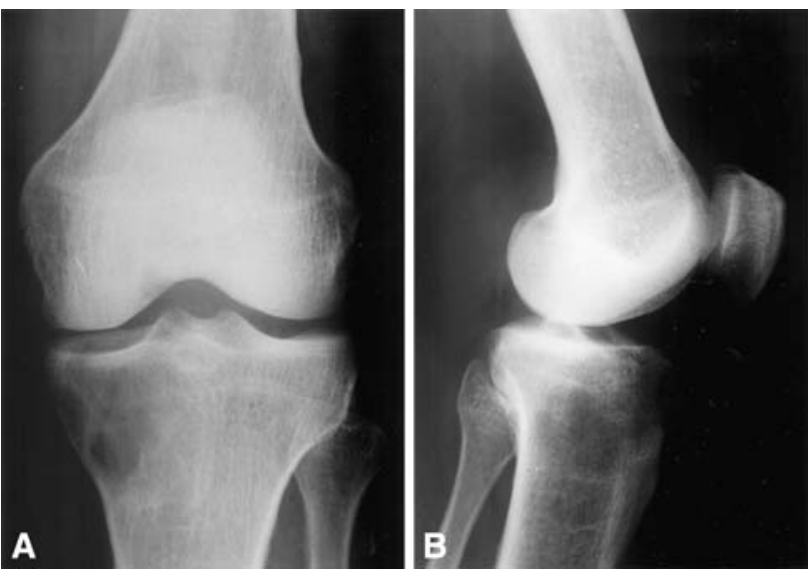

Fig. 1A-B Preoperative (A) anteroposterior and (B) lateral radiographs of the left knee show a lytic, geographic 1C lesion of the proximal medial tibia. Cortical thinning and extension to subchondral bone are evident, but there is no overt matrix production, periosteal reaction, or cortical breakthrough.
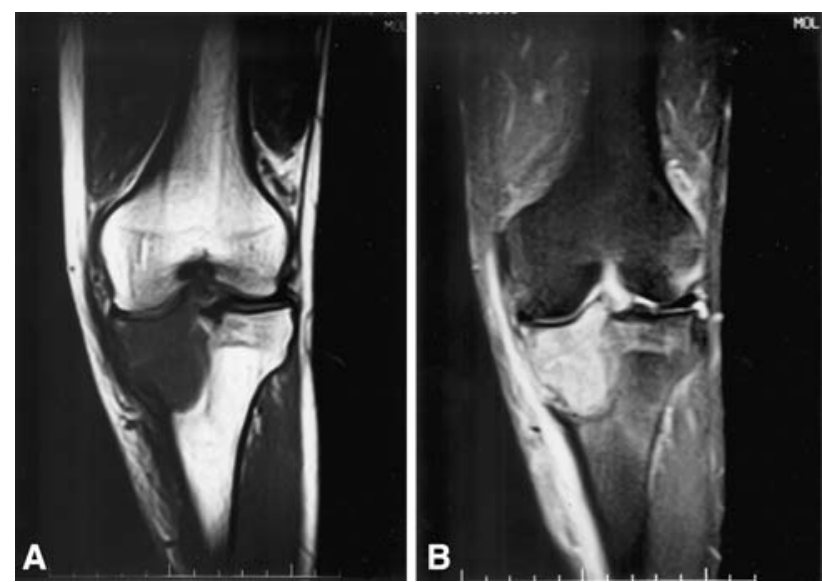

Fig. 2A-B Preoperative coronal (A) T1-weighted and (B) T2weighted STIR MR images show the lesion is mildly heterogeneous with hypointense to isointense signal versus muscle on the $\mathrm{T} 1$ images and increased signal versus fat on STIR imaging. There is extensive abnormal signal extending medially into the adjacent soft tissues, and mildly increased signal in the marrow adjacent to the lesion.

Langerhans cell histiocytosis

Plasmacytoma

Lymphoma

Osteosarcoma

Open biopsy with intraoperative frozen section analysis, extended curetting, phenolization, and polymethylmethacrylate cementation of the lesion were performed (Fig. 3). Histopathologic analysis was performed on the excised tissue (Fig. 4).

Based on the history and physical examination, imaging studies, and histologic appearance, what is the diagnosis and what further evaluation and/or treatments are indicated?
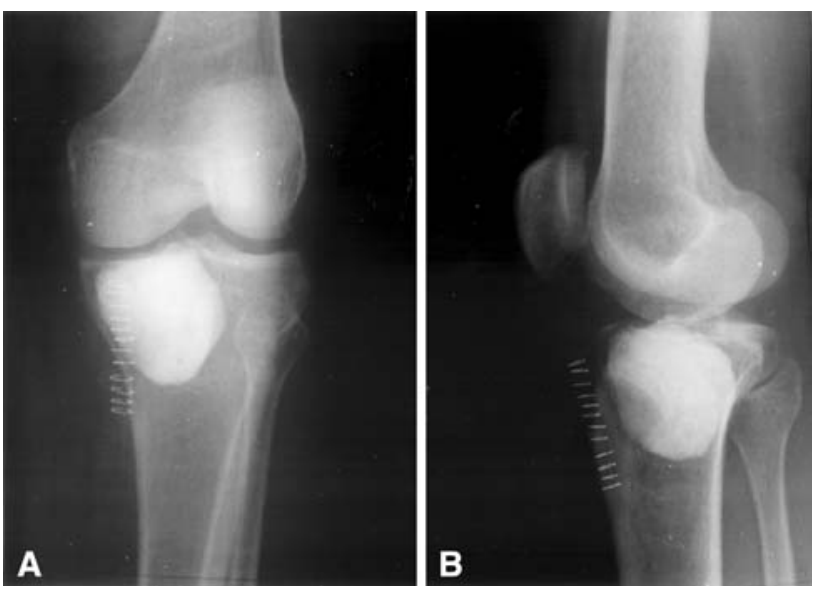

Fig. 3A-B Postoperative (A) anteroposterior and (B) lateral radiographs show the patient's knee after open biopsy, intraoperative frozen section analysis, extended curetting and phenolization, and polymethylmethacrylate packing of the lesion.

\section{Histology Interpretation}

The curetted specimen had numerous fragments of reddishbrown, hemorrhagic and gray, rubbery-appearing soft tissue measuring $6 \times 5 \times 1.1 \mathrm{~cm}$ in aggregate. Representative portions of the specimen were analyzed via frozen section and the remainder was submitted for permanent fixation in formalin, and gene rearrangement, flow cytometry, and immunohistochemical analysis. Microscopically, the lesion had a diffuse mixture of atypical lymphocytic and histiocytic cells. Nuclei were variable in shape with the predominant pattern being grooved or vesicular with prominent nucleoli. Glycogen was absent from the cytoplasm and the intercellular background had a complex reticulin framework. Flow cytometric analysis showed a B cell population that was CD19, CD20, and CD2 positive with kappa light-chain restriction (Fig. 4).

\section{Diagnosis}

Primary lymphoma of bone (large B cell type)

\section{Discussion and Treatment}

The eccentric, lytic intramedullary lesion extending from the metaphysis to subchondral bone suggested a differential diagnosis including giant cell tumor, benign fibrous histiocytoma, chronic osteomyelitis, Langerhans cell histiocytosis, plasmacytoma, lymphoma, and osteosarcoma.

The most likely diagnosis based on the lesion location, patient age, radiographic appearance, and tumor incidence was giant cell tumor of bone, which represents $15 \%$ of all 
Fig. 4A-C (A) Low-power (original magnification, $\times 200)$ and (B) high-power (original magnification, $\times 600)$ photomicrographs of the curetted specimen show atypical lymphocytes and histiocytic cells with grooved or vesicular nuclei and prominent nucleoli (Stain, hematoxylin and eosin). (C) The tissue stained diffusely positive for CD20 (original magnification, $\times 200$ ).
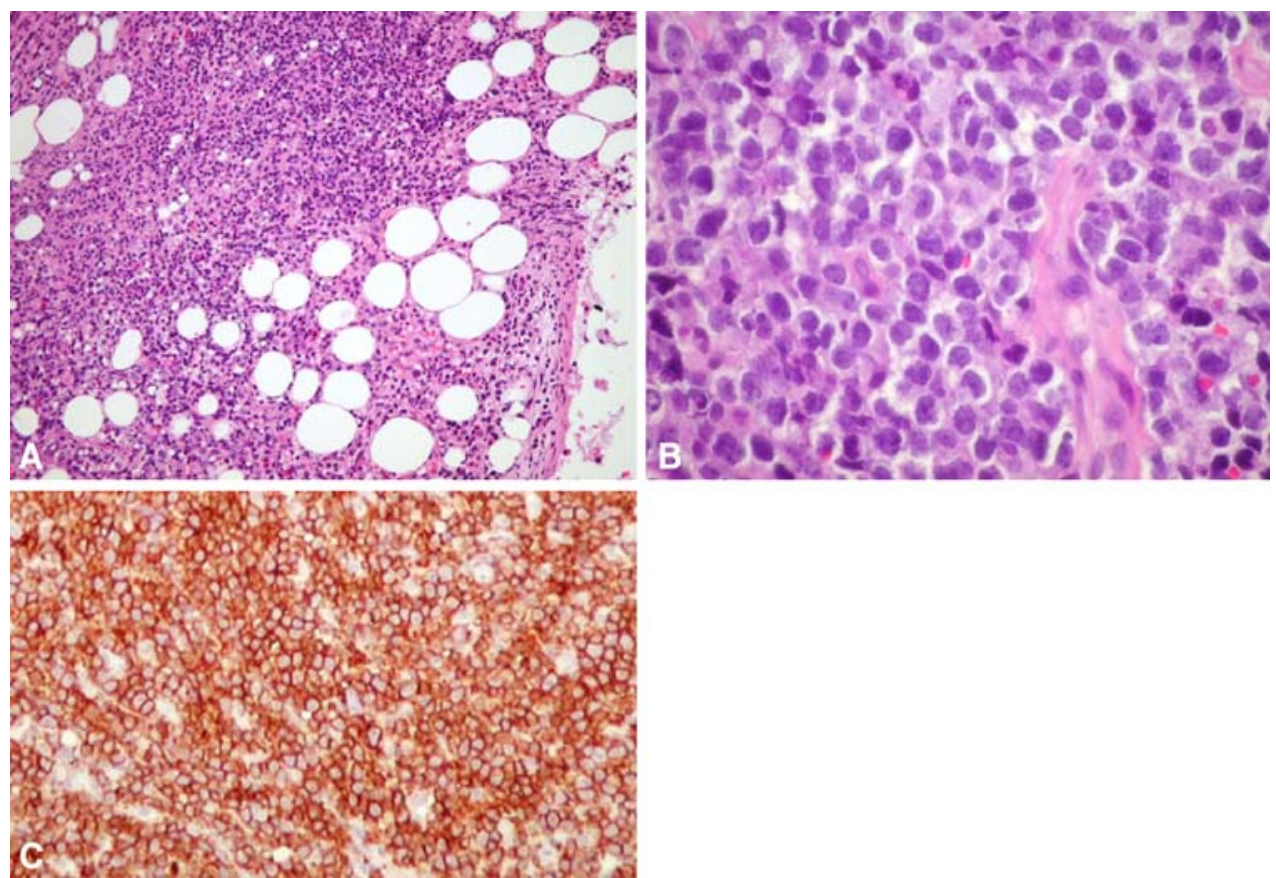

benign bone tumors, nearly $50 \%$ of which occur about the knee [19]. Giant cell tumors present most frequently in the second through fourth decades as purely nonmatrix-producing lytic lesions in the metaepiphysis, with a female predominance. Cortical thinning, as was evident radiographically in our patient, or destruction is common and advanced cases may present with a substantial soft tissue mass. The MRI findings in the current patient also were not inconsistent with this diagnosis, which was high on our differential diagnosis before biopsy and intraoperative frozen section analysis. Likewise, benign fibrous histiocytomas of bone can have a radiographic appearance and clinical presentation similar to giant cell tumor, but these lesions are rare and sometimes are considered regressive giant cell tumor despite the dissimilar histopathology [12].

Chronic osteomyelitis commonly presents as a painful, destructive lesion of bone. Langerhans cell histiocytosis, owing to its highly variable presentation and appearance, is included classically in the differential diagnosis for all cases in which infection is suspected. We considered infection and Langerhans cell histiocytosis relatively less likely in this instance owing to the absence of overt periosteal reaction, new bone formation, and the relatively small amount of intraosseous edema present on MRI. Both of these conditions also occur much more commonly in younger individuals, with approximately $3 / 4$ of eosinophilic granulomas occurring in patients younger than 20 years [17]. Langerhans cell histiocytoses that develop or persist in skeletally mature individuals may have a more aggressive clinical course [16]. Furthermore, our patient did not report any history of trauma, other known risk factors for osteomyelitis, or constitutional symptoms [8].

Solitary plasmacytoma of bone is a precursor lesion to multiple myeloma, with a transition rate of $65 \%$ to $100 \%$ at 15 years $[3,7]$. There is a $2: 1$ male predilection with a median age at presentation of 55 years, nearly a decade earlier than the typical presentation of myeloma [7]. Although our patient was relatively younger than typical for development of plasmacytomas, they frequently present as geographic, lytic lesions with variable cortical thinning, as occurred in our patient. The MRI findings in this case also are consistent with plasmacytoma. Therefore, plasmacytoma was a plausible consideration before the biopsy, although it was considered relatively less likely owing to our patient's age.

Osteosarcomas frequently occur about the knee and most commonly present in the second decade of life [2] but often are considered to have a bimodal age distribution. However, epidemiology data suggest osteosarcomas are actually more common in the fourth and fifth decades than in the sixth to eighth decades, with the former group having a prognosis that is intermediate between patients presenting earlier or later and a 5-year survival of approximately $50 \%$ [2]. On radiographs, most osteosarcomas have mineralization patterns suggestive of osteoblastic matrix or, in the case of telangiectatic osteosarcoma, predominant lysis of the bone with overt cortical destruction and expansion. Osteosarcoma was considered less likely in our patient because of the absence of periosteal reaction, soft tissue extension, and intralesional mineralization. 
Primary lymphoma of bone has been defined variably as lymphoma arising in the medullary cavity of one or multiple bones, without concurrent regional involvement of lymph nodes or visceral organs for at least 6 months. Some series therefore have restricted the diagnosis to patients presenting with an isolated lesion of bone, although the most common site of spread is to additional osseous sites [6]. Furthermore, exclusion of patients with greater than Stage IE disease may confer the false connotation of a localized process to what is, in terms of staging, relapse, and patient survival, a systemic disease; the variable inclusion and exclusion of patients with distant sites of osseous or other systemic disease at presentation make analysis of the outcomes for the disease difficult.

Primary lymphomas of bone account for approximately $3 \%$ to $7 \%$ of all primary osseous malignancies and extranodal lymphomas, with a slight male predilection [5]. The majority of these tumors are nonHodgkin's lymphomas, and as observed in our patient, as much as $92 \%$ are diffuse large B cell lymphomas (historically referred to as reticulum cell sarcomas) [6, 14]. Peak age at presentation is during the fifth decade, with most occurring after skeletal maturity [18]. Presenting complaints are generally a localized, dull, aching pain. As many as $50 \%$ of patients will present after 1 year or more of symptoms [4]. Patients are generally otherwise healthy, and systemic complaints or B symptoms are rare $[18,20]$. Physical examination findings are generally nonspecific. Palpable soft tissue masses may develop and adjacent joint effusions occasionally are encountered, although direct articular extension of tumor is rare.

The lesions are located in the lower extremity in $30 \%$ of cases, with the femur and pelvis representing the most common sites, followed by the spine and tibia $[6,11]$, with a predilection for the metadiaphyseal region. Radiographically, primary lymphomas of bone may have a blastic or mixed appearance. However, the lesions are purely lytic in as much as $70 \%$ of cases, without appreciable matrix production. Periosteal reaction is evident in approximately $1 / 2$ of cases and approximately $20 \%$ of patients present with an associated pathologic fracture [14]. Computed tomography findings generally show nonspecific lytic lesions but can be useful for evaluating cortical thinning and osseous integrity. Nuclear medicine studies generally show uptake equal to or exceeding that of the anterior-superior iliac spines but can be falsely negative in $2 \%$ to $4 \%$ of cases. MRI characteristics are variable but generally show an isointense to hyperintense muscle lesion on T1-weighted series, with relatively increased signal intensity relative to fat on $\mathrm{T} 2$-weighted images, as seen in our patient [14].

In addition to appropriate imaging of the primary lesion, staging via bone scan, computed tomography/positron emission tomography, and bone marrow biopsy should be completed before initiation of systemic treatment.
NonHodgkin's lymphomas are staged according to the Ann Arbor system [13], with Stage I disease referring to involvement of one lymph node region or extranodal (Stage IE) site. Stage II disease refers to involvement of two or more sites on the same side of the diaphragm, Stage III disease is involvement of sites on both sides of the diaphragm or splenic involvement (Stage IIIS), and Stage IV disease represents disseminated involvement of organs (eg, liver, lungs, or bone marrow). Although differing series have suggested stage may $[4,15]$ or may not $[6,9]$ be prognostic, identifying all sites of disease is essential in ensuring appropriate radiotherapeutic and chemotherapeutic treatment and/or monitoring patients for relapse. Slightly greater than $1 / 2$ of all patients with primary lymphomas of bone present with Stage IE disease [1, 4, 6, 9, 18].

Treatment regimens vary widely but generally consist of radiotherapy and chemotherapy for localized disease of the bone and chemotherapy with or without radiotherapy for advanced disease $[1,5,6,9,15]$. Overall response and local control rates may approach $94 \%$ to $100 \%$ regardless of treatment, and systemic relapse rates are higher with radiotherapy alone $[1,9,20]$. Surgery (apart from biopsy) generally has not been seen to impact prognosis and usually is reserved for overt or impending pathologic fractures, with resection and occasionally amputation necessary as salvage procedures for lesions unresponsive to chemotherapy and radiotherapy [9]. Our patient underwent intralesional treatment and polymethylmethacrylate stabilization to support the subchondral articular surface and prevent pathologic tibial plateau fracture.

The 5-year overall and disease-specific survival for primary lymphomas of bone have been reported consistently at approximately 60\% [4-6, 9, 15]. As noted, stage does not consistently correlate with prognosis and this apparent discrepancy may be attributable to the tendency for patients with more advanced stage disease to receive more aggressive systemic chemotherapy, versus lower-dose chemotherapy or radiotherapy alone for many patients with early-stage disease. Histologic subtype may be prognostic, with classic, large B cell, cleaved-nuclei-type primary lymphoma of bone having increased survival relative to the noncleaved-nuclei type, immunoblastic and pleomorphic lymphomas, or Hodgkin's lymphoma [4, 6, 9]. Advanced age at presentation ( $>40-60$ years) predicts higher diseasespecific mortality [6, 9].

Our patient was referred to a hematology/oncology specialist for staging and found to have isolated, Stage IE disease. He was treated with six cycles of CHOP (cyclophosphamide, doxorubicin, vincristine, and prednisone) chemotherapy followed by adjuvant external beam radiation therapy of 45 Gy to the left leg using a shrinking field technique. The patient responded well to treatment and has been continuously disease-free for 74 months since 
completing his adjuvant therapies (81 months postoperatively). At last followup, he had no local or systemic complaints, with full, painless function of his left lower extremity and normal knee range of motion.

We present a case of primary lymphoma of bone with a somewhat unusual radiographic presentation in a juxtaarticular location, mimicking a giant cell tumor of bone. Adherence to appropriate biopsy principles, including obtaining adequate tissue through an extensile approach and use of intraoperative frozen section, and consultation with an experienced musculoskeletal pathologist were critical in achieving the correct diagnosis in this case. Although the operative treatment was somewhat more aggressive than that typically required for primary lymphoma of bone, which was suspected based on frozen section analysis, we believed intralesional treatment and polymethylmethacrylate stabilization were indicated to prevent a pathologic fracture of the involved tibial plateau, and our patient experienced immediate relief of his local symptoms postoperatively. Ultimately, a good functional outcome and, at present, long-term survival without local or systemic relapse of disease was achieved in this patient through appropriate referrals for aggressive multimodal systemic and local adjuvant therapies.

\section{References}

1. Baar J, Burkes RL, Bell R, Blackstein ME, Fernandes B, Langer F. Primary non-Hodgkin's lymphoma of bone. Cancer. 1994;73: 1194-1199.

2. Damron TA, Ward WG, Stewart A. Osteosarcoma, chondrosarcoma, Ewing's sarcoma: National Cancer Data Base Report. Clin Orthop Relat Res. 2007;459:40-47.

3. Dimopoulos MA, Kiamouris C, Moulopoulos LA. Solitary plasmacytoma of bone and extramedullary plasmacytoma. Hematol Oncol Clin North Am. 1999;13:1249-1257.

4. Durr HR, Muller PE, Hiller E, Maier M, Baur A, Jansson V, Refior HJ. Malignant lymphoma of bone. Arch Orthop Trauma Surg. 2002;122:10-16.
5. Hameed M. Small round cell tumors of bone. Arch Pathol Lab Med. 2007;131:192-204.

6. Heyning FH, Hogendoorn PC, Kramer MH, Hermans J, KluinNelemans JC, Noordijk EM, Kluin PM. Primary non-Hodgkin's lymphoma of bone: a clinicopathological investigation of 60 cases. Leukemia. 1999;13:2094-2098.

7. $\mathrm{Hu} \mathrm{K}$, Yahalom J. Radiotherapy in the management of plasma cell tumors. Oncology. 2000;14:101-108.

8. Lazzarini L, Mader JT, Calhoun JH. Current Concepts Review: Osteomyelitis in long bones. J Bone Joint Surg Am. 2004;86: $2305-2318$

9. Lewis VO, Primus G, Anastasi J, Doherty D, Montag AG, Peabody TD, Simon MA. Oncologic outcomes of primary lymphoma of bone in adults. Clin Orthop Relat Res. 2003;415: 90-97.

10. Lodwick GS, Wilson AJ, Farrell C, Virtama P, Dittrich F. Determining growth rates of focal lesions of bone from radiographs. Radiology. 1980;134:577-583.

11. Marshall DT, Amdur RJ, Scarborough MT, Mendenhall NP, Virkus WW. Stage IE primary non-Hodgkin's lymphoma of bone. Clin Orthop Relat Res. 2002;405:216-222.

12. Matsuno T. Benign fibrous histiocytoma involving the ends of long bone. Skeletal Radiol. 1990;19:561-566.

13. Moormeier JA, Williams SF, Golomb HM. The staging of nonHodgkin's lymphomas. Semin Oncol. 1990;17:43-50.

14. Mulligan ME, McRae GA, Murphey MD. Imaging features of primary lymphoma of bone. AJR Am J Roentgenol. 1999;173: 1691-1697.

15. Ostrowski ML, Unni KK, Banks PM, Shives TC, Evans RG, O'Connell MJ, Taylor WF. Malignant lymphoma of bone. Cancer. 1986;58:2646-2655.

16. Plasschaert F, Craig C, Bell R, Cole WG, Wunder JS, Alman BA. Eosinophilic granuloma: a different behaviour in children than in adults. J Bone Joint Surg Br. 2002;84:870-872.

17. Schajowicz F, Slullitel J. Eosinophilic granuloma of bone and its relationship to Hand-Schuller-Christian and Letterer-Siwe Syndromes. J Bone Joint Surg Br. 1973;55:545-565.

18. Stein ME, Kuten A, Gez E, Rosenblatt KE, Drumea K, Ben-Shachar M, Zidan J, Haim N, Epelbaum R. Primary lymphoma of bone: a retrospective study. Oncology. 2003;64: 322-327.

19. Szendroi M. Giant-cell tumour of bone. J Bone Joint Surg Br. 2004;86:5-12.

20. Zinzani PL, Carrillo G, Ascani S, Barbieri E, Tani M, Paulli M, Stefoni V, Sabattini E, Alinari L, Binazzi R, Tura S, Baccarani M, Pileri SA. Primary bone lymphoma: experience with 52 patients. Haematologica. 2003;88:280-285. 